JING QIAO, Ph.D. ${ }^{1}$

E-mail: qiaojing55@126.com

LISHAN SUN, Ph.D. ${ }^{1}$

(Corresponding author)

E-mail: Issun@bjut.edu.cn

SHI QIU, Ph.D. ${ }^{1}$

E-mail: qshi7777@gmail.com

JIAN RONG, Ph.D. ${ }^{1}$

E-Mail: jrong@bjut.edu.cn

XIAOMING LIU, M.S. ${ }^{1}$

E-mail: liuxm@bjut.edu.cn

${ }^{1}$ Beijing Key Laboratory of Traffic Engineering,

Beijing University of Technology, 100 Pingleyuan,

Chaoyang District, Beijing, 100124, China
Traffic Engineering

Preliminary Communication

Submitted: 19 May 2016

Accepted: 7 Apr. 2017

\title{
REDUCING BIDIRECTIONAL PEDESTRIAN CONFLICT BASED ON LANE FORMATION PHENOMENON IN SUBWAY CORRIDORS
}

\begin{abstract}
With the rapid increase of the subway passenger volume, the conflict among passengers emerges as a significant issue which affects subway serviceability, especially in the bidirectional flow. The aim of this study is to explore the characteristics of the bidirectional flow of pedestrians in a subway corridor. Pedestrian experiments were conducted to investigate microscopic characteristics of the pedestrian flow. It was found that the microscopic characteristics, including the walking speed and turning angle, were time-dependent and had a generalized trend with time. It was also found that different pedestrian volumes affected the microscopic characteristics. Based on the trend of the microscopic characteristics, the lane formation phenomenon was observed and quantitatively studied, identifying three phases: conflict phase, lane formation phase, and steady lane phase. To alleviate the bidirectional pedestrian conflict, additional pedestrian experiments for the countermeasure of adding separating strap in the corridor, which was based on the lane formation analysis, was conducted. The effectiveness of the countermeasure was demonstrated through a before-and-after comparison. The results showed that adding the separation between the adjacent lanes had the best performance in reducing the conflicts. The results would provide a rationale for subway managers in optimizing the corridor bidirectional pedestrian flow.
\end{abstract}

\section{KEY WORDS}

rail transit; bidirectional flow; pedestrian experiment; countermeasures;

\section{INTRODUCTION}

With the massive construction and new opening to traffic, the average daily passenger traffic (ADPT) of the subway has increased quickly, especially in Beijing, and has exceeded 10 million since 2013 [1]. The rapid growth of passengers has brought unprecedented challenges to subway management. According to the research team questionnaire survey, $83 \%$ of passengers, which was 410 passengers out of 494 valid surveyed, perceived uncomfortable or stressful riding the subway during rush hours. The large crowd is the most significant factor affecting passengers' comfort. Hence, reducing the passenger conflict in subway has become an immediate concern of the researchers and practitioners.

Pedestrian flows can be observed in many traffic systems and there appear spatial patterns characterized in common [2-3]. To improve pedestrian flow organization the first step is to characterize the pedestrian behaviour in a reasonable and scientific manner. The analysis of pedestrian flow is fundamental in massive transit planning, facility design, and operations management. Although the study of pedestrian traffic was initiated in the 1950s, which was later than the first research of auto traffic, it has developed fast over the recent years with the advance of modern data collection technologies [4-8].

With the theoretical and methodical analysis of pedestrian flow, the bidirectional flow, which is a typical pedestrian flow organization, has attracted substantial attention [8-13]. The interactions of bidirectional pedestrian streams would induce a serious conflicting effect [14-15]. In bidirectional flows, pedestrians tend to follow their predecessors who move toward the same direction and consequently two or more lanes are formed [16]. A few studies have been conducted to analyse the bidirectional flow. For example, Lam et al. [17] analysed and calibrated individual behaviour characteristics of a two-way pedestrian flow at signalized intersections. They also identified that 
bidirectional flow conflict was affected by speed and capacity [18]. Additional studies focused on specific bidirectional characteristics such as lane numbers and selection probabilities [11-12]. The conventional studies, however, mostly targeted normal open-space facilities, such as intersection crosswalks or pedestrian sidewalks, and lacked a qualitative analysis in lane formed and control managements.

In subway system, the corridor is one of most commonly used walking facilities for passenger transfer. It is usually an underground pedestrian passageway where pedestrians walk from one end to the other. Regarding pedestrian flow, the bidirectional flow is the most typical form of the subway system. From the research team field survey, the typical bidirectional pedestrian flow ratio on a regular and frequent basis during rush hours at Beijing rail station was 1:1 (50\% of pedestrian flow in one direction, and $50 \%$ in the other direction), when the conflicting phenomenon was observed.

The objective of this paper was to investigate the microscopic characteristics of bidirectional pedestrian flow and explore the method to reduce the pedestrian conflict using countermeasures. Pedestrian experiments were conducted in this research. The specific self-organization phenomenon of bidirectional conflict in the corridor was quantitatively studied with the microscopic parameters of pedestrian behaviour extracted from the video footages. By analysing the change of mean speed and turning angle along with time, the pattern of lane formation was identified. Then, the methods to reduce the pedestrian conflict were evaluated through experiments. The before-and-after analysis was applied to demonstrate the effectiveness of the countermeasures.

\section{DESIGN OF PEDESTRIAN EXPERIMENT}

To analyse the bidirectional flow in a subway corridor, data collected from real-world would be preferred. Due to the complex condition such as low ceilings and uncontrollable traffic volume at the subway station, it is extremely difficult and unsafe to take and analyse the video footage directly. The pedestrian experiment as an effective approach in analysing pedestrian walking behaviour has been introduced in this study [1921]. The basic procedures of a pedestrian experiment are as follows: a similar setting to real-world conditions based on the research objectives is set up; walking pedestrians are videotaped; the video footages are used for deriving macroscopic and microscopic characteristics. The advantage of the pedestrian experiment includes controllable circumstances and strong purpose-oriented setup (i.e., experimental settings are basic but fitted to the needs) [22-23]. Therefore, this study also used the pedestrian experiments to investigate the bidirectional conflict at the subway corridor.

\subsection{Experiment organization}

A series of pedestrian experiments were conducted on the courtyard of the Beijing University of Technology (BJUT) in the afternoon of 17 April 2015. In total, 50 healthy undergraduate students were selected from the university as experiment participants. The male and female students ratio was 1:1. To minimize the acquaintances among the students, which might have influenced the results, the students were selected from different classes and departments. The age of the participants ranged from 19 to 23 years. The average height was $1.68 \mathrm{~m}$ with a standard deviation of 8.11 $\mathrm{cm}$. According to the field survey, in Beijing subway at rush-hour, there are mainly three luggage types that are: none, small luggage, and medium luggage with the average frequency percentages 37\%, 44\%, and $19 \%$, respectively. Thus, the participants were asked to carry luggage or handbags per their preference in each experiment to simulate real pedestrian flow conditions. The actual experimental scene is shown in Figure 1a.

According to the definition in TRB Transit Cooperative Research Program Report, the standard bidirectional passageway capacity is 4,000 person $/ \mathrm{h} / \mathrm{m}$ [24]. In this study, considering traffic conflict may vary under different volumes, five levels of the pedestrian volumes were investigated: 3,000 person/h/m, 3,500 person/h/m, 4,000 person/h/m, 4,500 person/h/m and 5,000 person $/ \mathrm{h} / \mathrm{m}$. In the experimental configuration, the pedestrian flow ratio was 1:1; two groups of participants, of which 25 in red hats and 25 in black hats, stood at 5 metres away from each side of the entrance. To achieve the 3,000, 3,500, 4,000, 4,500 and 5,000 person/h/m pedestrian volumes effectively, the participants stood as rows in each group, each of which had averages of 1.3, 1.5, 1.7, 1.9 and 2.1 experiment participants, in the preparation zone. After the signal of instruction was on, the participants started to walk in the random and natural state within the preparation zone.

For each volume, two trials were performed. The rules and purposes of the experiment were explained to the participants through two pre-experiments. Before each trial, all participants were randomly divided into two equal groups. The starting positions of the participants were randomized in front of the yellow start line for each trial, which was intended to make each participant face various situations. This randomization would also prevent the learning behaviour from happening between the trials. The hat colours of the two groups were used to distinguish the two-way traffic for automated data processing.

\subsection{Establishing experiment scene}

Differing from conventional pedestrian experiments, specific experimental settings were tailored for this study to reproduce the actual subway corridor 


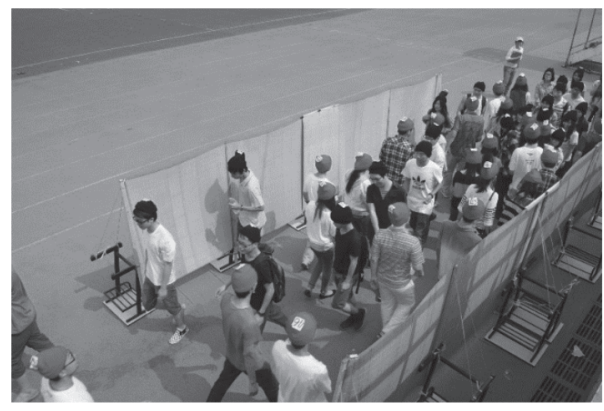

a) Actual experimental scene

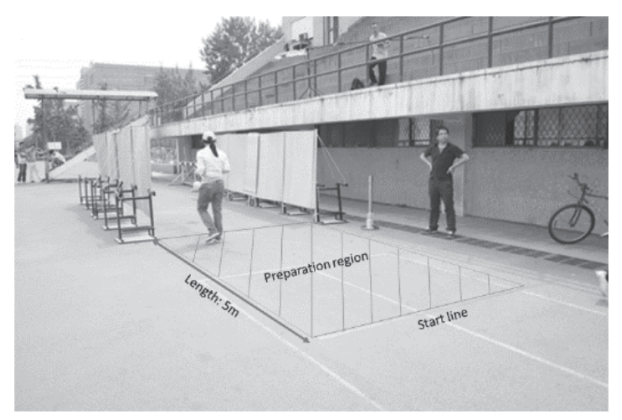

c) Preparation zone

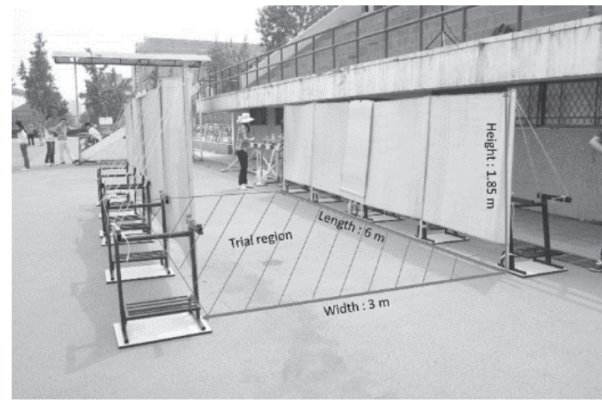

b) Analysis zone

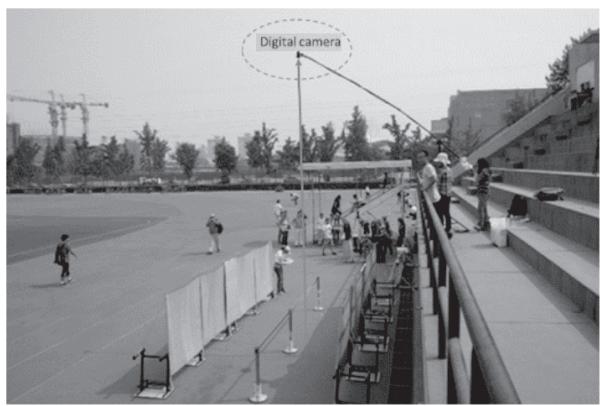

d) Digital camera setting

Figure 1 - Experimental scene

circumstances. The width of the subway corridor usually ranges from $3 \mathrm{~m}$ to $6 \mathrm{~m}$. Thus, the experimental corridor was $6 \mathrm{~m}$ long and $3 \mathrm{~m}$ wide with two outlets for a pedestrian entering and exiting. Two vertical boards were elicited to mimic the walls of the subway corridor. To make the experiment participants mentally perceive an enclosed space, the height of the board wall was $1.85 \mathrm{~m}$, which was higher than the average Chinese stature $1.71 \mathrm{~m} \mathrm{[25].}$

To ensure a constant environment, the experiment was conducted on the playground of the Beijing University of Technology (BJUT) at noon of 21 May 2013, when the ambient conditions were favourable (overcast, reasonably constant light intensity). The detail of analysis zone is shown in Figure $1 b$. To obtain the normal flow without being affected by a starting delay, two start lines $5 \mathrm{~m}$ away from each outlet were drawn, as shown in Figure 1c. Thus, it was assumed that the flow entering the corridor was a normal flow.

To analyse the pedestrian behaviour in an automated fashion, a digital camera was placed in the middle of the corridor $6 \mathrm{~m}$ high perpendicular to the ground, as highlighted in Figure 1d. The digital camera had a resolution of 1,920x1,080 pixels and a frame capture rate of 50 frames per second, adequate for analysing the speed and position of pedestrians [26].

By analysing the pedestrian experiment video footages, the pedestrian parameters were derived through an automotive identifying arithmetic for detecting objects, which combines the Gaussian Mixture Model (GMM), the K-means clustering on colour features, and a target tracking method based on Lucas-Kanade
(LK) [27]. To verify the accuracy of automotive identifying arithmetic, trial experiments were conducted before the formal experiments, showing that almost all pedestrians participating in the experiment could be detected and tracked. For the data of micro parameters (speed and turning angle), the difference between the automotive identifying arithmetic and the manual identifying arithmetic were analysed using ANOVA, showing no significant difference (Table 1). This verified the automotive identifying arithmetic for the acquisition of pedestrian parameters data.

Table 1 - Comparison of automotive identifying arithmetic and manual methods

\begin{tabular}{||c|c|c|c||}
\hline \hline Sequence & $\begin{array}{c}\text { Volume } \\
{[\mathrm{p} / \mathrm{h} / \mathrm{m}]}\end{array}$ & $\begin{array}{c}\text { Sig. of turning } \\
\text { angle }\end{array}$ & $\begin{array}{c}\text { Sig. of } \\
\text { speed }\end{array}$ \\
\hline \hline 1 & 3,000 & 0.04 & 0.08 \\
\hline 2 & 3,500 & 0.10 & 0.11 \\
\hline 3 & 4,000 & 0.12 & 0.17 \\
\hline 4 & 4,500 & 0.17 & 0.19 \\
\hline 5 & 5,000 & 0.21 & 0.22 \\
\hline
\end{tabular}

\section{ANALYSIS OF BIDIRECTIONAL LANE FORMATION PHENOMENON}

In this section, the microscopic parameters defined for conflict analysis, such as travel speed and weaving behaviour of the pedestrians, were extracted from the video footages in the pedestrian experiments to analyse bidirectional corridor flow under different volumes. 
With the automated extraction of the microscopic parameters, the statistics of the parameters were used to characterize the lane formation phenomenon.

\subsection{Definition of evaluation parameters}

A prerequisite for conflict analysis is to measure microscopic parameters for characterizing pedestrian behaviour. In this study, the two commonly used microscopic parameters, the walking speed $(v)$ and the turning angle $(\theta)$, were extracted for the pedestrian conflict analysis [26]. The walking speed ( $v)$, a basic parameter for describing the pedestrian flow, is the walking distance to a unit of time. Additionally, this study invents the concept of turning angle $\Delta \theta_{i}$, the angular difference between time $i-1\left(t_{i}-1\right)$ and time $i\left(t_{j}\right)$, to analyse the characteristic of the weaving behaviour, as shown in Equation 1:

$$
\Delta \theta_{i}=\arctan \frac{y_{i+1}-y_{i}}{x_{i+1}-x_{i}}-\Delta \theta_{i-1} \quad\left(\Delta \theta_{i}>0\right)
$$

where $\Delta \theta_{i}$ is the turning angle in $t_{i} ; t_{i}$ is pedestrian travel time in corridor $(\mathrm{s}) ; x_{i}$ is the pedestrian lateral displacement in $t_{i}(\mathrm{~m})$; and $y_{i}$ is the pedestrian longitudinal displacement in $t_{i}(\mathrm{~m})$. The time slice for speed and turning angle calculation is $0.02 \mathrm{~s}$ [26]. The turning angle is a plus-minus number; it is recorded as a positive number when pedestrian turns left, and, otherwise, it is recorded as a negative number.

\subsection{Analysis of speed and turning angle}

The speed of each pedestrian in the experiment was obtained through the video footage analysis. Table 2 summarizes the descriptive statistics of speed under different volumes for the entyre group. Figure 2 shows the change of mean and standard deviation of the speed with the travel time for different volume of pedestrians. The mean speed is a global measure of the group pedestrian behaviour. The standard deviation is a variation measure which indicates the performance of individual pedestrians caused by the conflict.

As shown in Figure 2, the mean speed decreased in the initial phase of the experiment and then increased after a certain point. The standard deviation of speed showed an opposite trend; the standard deviation increased initially and then decreased with the time increase, despite a slight fluctuation. For all pedestrian volumes, the same trend was observed.

The above observations can be explained via considering the real-world circumstance, that is, the pedestrian flow as a high-speed free flow entering a subway corridor. While the pedestrians encounter each other, the mean speed is decreased due to the

Table 2 - Descriptive statistics of speed under different volumes

\begin{tabular}{|c|c|c|c|c|c|c|}
\hline \multicolumn{2}{|c|}{ Volume [person/h/m] } & 3,000 & 3,500 & 4,000 & 4,500 & 5,000 \\
\hline \multicolumn{2}{|l|}{ Mean [m/s] } & 1.24 & 1.13 & 1.09 & 1.07 & 0.89 \\
\hline \multicolumn{2}{|c|}{ Median [m/s] } & 1.24 & 1.14 & 1.09 & 1.06 & 0.87 \\
\hline \multicolumn{2}{|c|}{ Std. deviation [m/s] } & 0.15 & 0.16 & 0.2 & 0.21 & 0.25 \\
\hline \multicolumn{2}{|c|}{ Minimum [m/s] } & 0.89 & 0.67 & 0.58 & 0.5 & 0.38 \\
\hline \multicolumn{2}{|c|}{ Maximum $[\mathrm{m} / \mathrm{s}]$} & 1.59 & 1.53 & 1.7 & 1.66 & 1.57 \\
\hline \multirow{3}{*}{ Percentiles [m/s] } & $25 \%$ & 1.14 & 1.01 & 0.97 & 0.92 & 0.7 \\
\hline & $50 \%$ & 1.24 & 1.14 & 1.09 & 1.06 & 0.87 \\
\hline & $75 \%$ & 1.33 & 1.24 & 1.22 & 1.2 & 1.07 \\
\hline
\end{tabular}
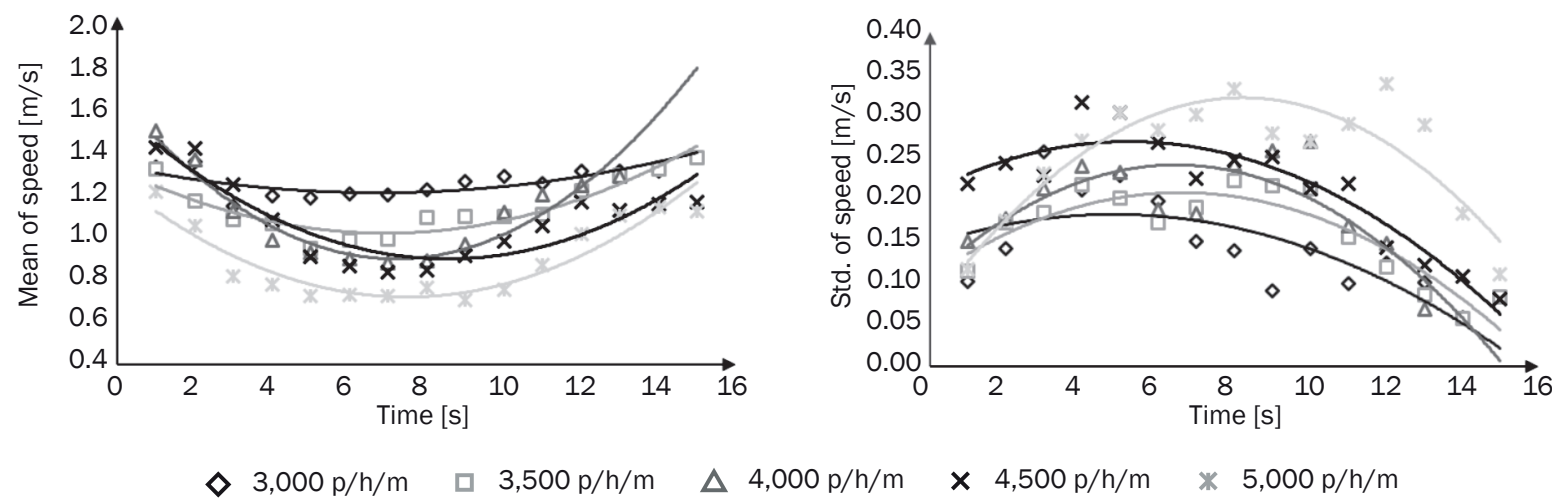

$3,000 \mathrm{p} / \mathrm{h} / \mathrm{m} \quad \mathrm{Z}, 500 \mathrm{p} / \mathrm{h} / \mathrm{m}$

$4,000 \mathrm{p} / \mathrm{h} / \mathrm{m}$

$\times \quad 4,500 \mathrm{p} / \mathrm{h} / \mathrm{m}$

* $5,000 \mathrm{p} / \mathrm{h} / \mathrm{m}$

a) Mean speed with time

b) Std. speed with time

Figure 2 - Speed (Mean and Standard deviation) of different volumes with time 
"obstacles" in the way under bidirectional flow. The standard deviation, however, increases as the "obstacles" affected pedestrians at different locations of the corridor differently. Those who enter the corridor first, encounter more conflicts, and the followers may change speed subsequently. According to the classic self-organization theory, the pedestrians would walk in separate lanes which may have been formed after the initial conflict [28]. This explains why the speed rises and standard deviation drops after a certain point. Based on the self-organization phenomenon, the lane formation is likely to occur around the time when the lowest mean speed is exhibited.

Also, traffic volume is related closely with the mean and standard deviation of speed. The average pedestrian speed was decreased with the increase of traffic volume. The average speed at volume 5,000 person $/ \mathrm{h} / \mathrm{m}$ was $0.89 \mathrm{~m} / \mathrm{s}$, much lower than the speed at 3,000 person $/ \mathrm{h} / \mathrm{m}$, which was $1.23 \mathrm{~m} / \mathrm{s}$. The standard deviation of speed showed a similar pattern, while the high volume of which was more significant than that of the low volume. The speed rising and standard deviation declining occurred later when the pedestrian volume was higher.

Despite that the turning angles were plus-minus numbers, the absolute value of the turning angles was calculated to analyse the descriptive statistics.
The standard deviation of the turning angles, however, were determined using the plus-minus numbers, so as to illustrate the degree of dispersion in the left and right angles. Table 3 summarizes the descriptive statistics of the turning angle under different volumes for the entyre group. Figure 3 presents the relationship between the mean and standard deviation of the turning angle and the travel time.

The statistics analysed in the speed section showed the same characteristics in the turning angle parameters. The mean and standard deviation of the turning angle were similar to the observations of the speed parameter: the mean angle increased first and then decreased. The standard deviation decreased after an initial increase. The self-organization phenomenon was observed in the statistics and trends of the turning angle parameter. On the traffic volume, the turning angle increased with the volume increase. The average turning angle at volume 5,000 person $/ \mathrm{h} / \mathrm{m}$ was 8.86 , which was much higher than the turning angle of 3,000 person $/ \mathrm{h} / \mathrm{m}$. Since the turning angles in higher volumes were larger, there is an apparent longer lasting of conflict. Furthermore, the extent and range of the standard deviation expanded with the rising of flows. A possible theory to account for this phenomenon is the least effort in psychology [29]. In this theory, all pedestrians are unwilling to change or

Table 3 - Descriptive statistics of turning angle under different volumes

\begin{tabular}{|c|c|c|c|c|c|c|}
\hline \multicolumn{2}{|c|}{ Volume [person/h/m] } & 3,000 & 3,500 & 4,000 & 4,500 & 5,000 \\
\hline \multicolumn{2}{|c|}{ Mean [m/s] } & 5 & 5.27 & 7.31 & 6.08 & 8.86 \\
\hline \multicolumn{2}{|c|}{ Median [m/s] } & 4.23 & 4.43 & 5.62 & 4.82 & 6.77 \\
\hline \multicolumn{2}{|c|}{ Std. deviation [m/s] } & 3.8 & 4.14 & 6.12 & 5.53 & 9.11 \\
\hline \multicolumn{2}{|c|}{ Minimum [m/s] } & 0.2 & 0.1 & 0.18 & 0.11 & 0.18 \\
\hline \multicolumn{2}{|c|}{ Maximum $[\mathrm{m} / \mathrm{s}]$} & 15.93 & 19.05 & 28.89 & 32.35 & 53.84 \\
\hline \multirow{3}{*}{ Percentiles [m/s] } & $25 \%$ & 2 & 1.96 & 2.63 & 2.26 & 3.2 \\
\hline & $50 \%$ & 4.23 & 4.43 & 5.75 & 4.82 & 6.77 \\
\hline & $75 \%$ & 7.07 & 7.7 & 10.84 & 8.62 & 11.38 \\
\hline
\end{tabular}
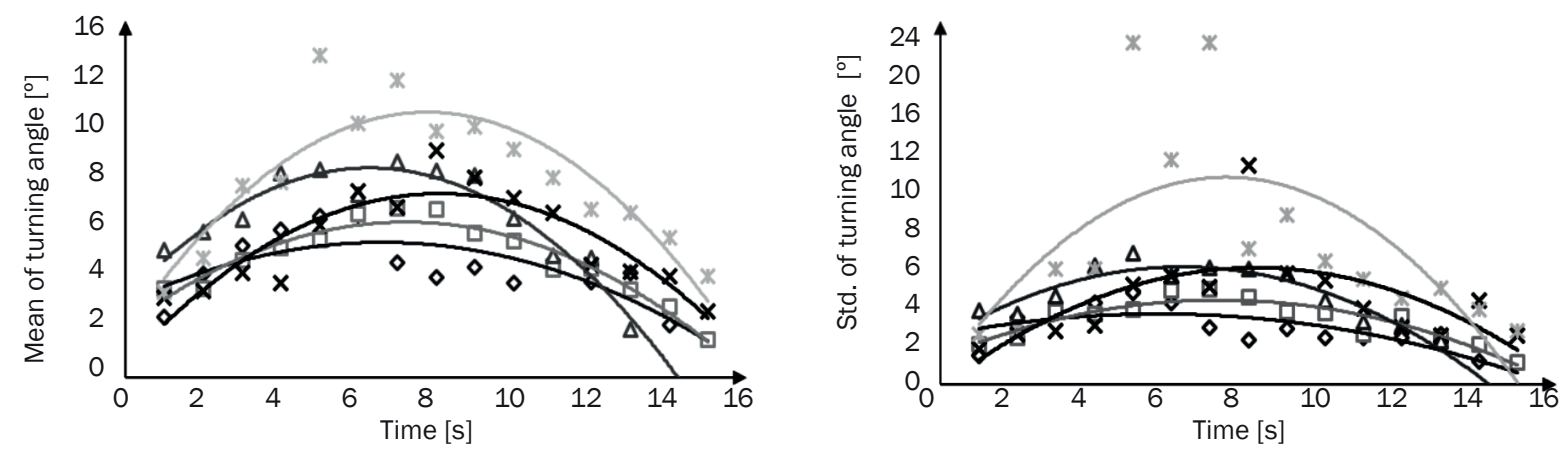

$\diamond 3,000 \mathrm{p} / \mathrm{h} / \mathrm{m} \quad \square 3,500 \mathrm{p} / \mathrm{h} / \mathrm{m} \quad \Delta 4,000 \mathrm{p} / \mathrm{h} / \mathrm{m}$

$\times 4,500 \mathrm{p} / \mathrm{h} / \mathrm{m} \quad * 5,000 \mathrm{p} / \mathrm{h} / \mathrm{m}$

a) Mean turning with time

b) Std. turning with time

Figure 3 - Turning (Mean and Standard deviation) of different volume with time 
compromise their optimal and comfortable route (i.e. keep straight). With the increase in the volume, the impact of this unwillingness is enlarged, and the conflict is more severe.

\subsection{Quantifying three phases of lane formation}

As introduced by Helbing [20], the walking pedestrian self-organization is created by individuals' constant attempt to improve their situation. In the context of bidirectional walking, the self-origination refers to the phenomenon that pedestrians tend to follow the same directional flow and thereby multiple lanes are formed in the process $[16,30]$. Although the process of lane formation is an important phenomenon, attracting considerable attention, it has been only qualitatively studied in the past. For example, Helbing et al. [20] found that the lane forming process was dynamic; the hierarchical position and layers would change. In this study, the lane process was quantitatively analysed with the parameters extracted from the pedestrian experiments.

To further investigate the process of lane formation, this study analysed the change of speed and turning angle along the experiment process. A second order polynomial fitting was applied to both speed and turning angle data set. For most of the scenarios, the coefficient of determination $\left(R^{2}\right)$ is high. Table 4 lists the extreme values of the polynomials obtained through the first derivation. Even though the extreme points for both speed and turning angle increase with the increase of pedestrian volumes, the extreme points were close to each other, which implied that a potential break existed in the self-organization and a higher flow would lead to more conflicts. The states of the pedestrian locations for three different time points (one second before the extreme point, the extreme point, and one second after the extreme point) were extracted, as shown in Figure 4. The conflict gradually decreased. At the first column of Figure 4 (the locations before the extreme point), weavings appeared between the pedestrians from the two directions; at the second column, there were fewer weavings; in the last column (the locations after the extreme time point), the lanes were formed except the figures for the volume 5,000 where few conflicts still existed. Therefore, the three phases of lane formation can be characterized as follows:
1) Conflict phase. At the beginning of this phase, the entering pedestrians possess free flow speed. With the encountering of the opposite-directional flow, the pedestrians who enter the corridor first encounter more conflict, and the followers may change speed and walking direction subsequently, resulting in the decrease of mean speed and the increase of mean turning angle. According to the data, this phase usually appears within the first 6 seconds of the experiment.

2) Lane formation phase. The breakpoint occurs in this phase. According to the self-organization theory, which is based on the principle of least effort in psychology [29], all pedestrians aim to minimize their predicted disutility of walking the optimal choice; i.e. following closely behind some other person who moves in the same direction. The emerging lanes in this phase are unsteady; they may disappear, merge or split again. Overall, $6-8 \mathrm{~s}$ is the range of the lane formation completion.

3) Steady lane phase. The pedestrians would walk in the separate lanes, which may have been formed after the initial conflict [20,31]. The followers are prone to follow the predecessors in the lane. This explains why the speed rises and standard deviation drops after a certain point. Based on the self-organization phenomenon, the lane formation is likely to occur around the time when the lowest mean speed is exhibited.

To further explore the characteristics of the lane, lane patterns and lane widths were quantitatively analysed. As shown in Figure 4, two, three, four, and five lanes appeared in different runs of experiments. Several lanes contained a volatile and brief feature; i.e. the lane exhibits a broken state during lane formation phase. Based on quantitative analysing the characteristics of the three phases of lane formation, the two lanes and four lanes had the features of the steady lane phase and, thus were defined as steady lanes, as shown in Figure 5. Different colours indicated the different direction lanes in the bidirectional pedestrian flow. It was found that an odd number of lanes exhibited a broken state and an even number of lanes exhibited a steady state. Note that pedestrians in the experiments were largely right-hand-sided, typical for countries in which traffic regulations stipulate walking on the right side of the street [21].

Table 4 - The threshold time of lane-forming [s]

\begin{tabular}{|c|c|c|c|c|c||}
\hline Volume $[\mathrm{p} / \mathrm{h} / \mathrm{m}]$ & Speed (Mean) & Speed (Std.) & Angle (Mean) & Angle (Std.) & Mean \\
\hline \hline 3,000 & 7.83 & 5.66 & 6.6 & 5.66 & 6.44 \\
\hline 3,500 & 7.9 & 7.71 & 7.23 & 7.25 & 7.55 \\
\hline 4,000 & 7.55 & 6.81 & 6.27 & 6.28 & 6.73 \\
\hline 4,500 & 8.57 & 7.2 & 8.08 & 8.197 & 8.01 \\
\hline 5,000 & 8.48 & 9.13 & 7.77 & 7.42 & 8.20 \\
\hline
\end{tabular}



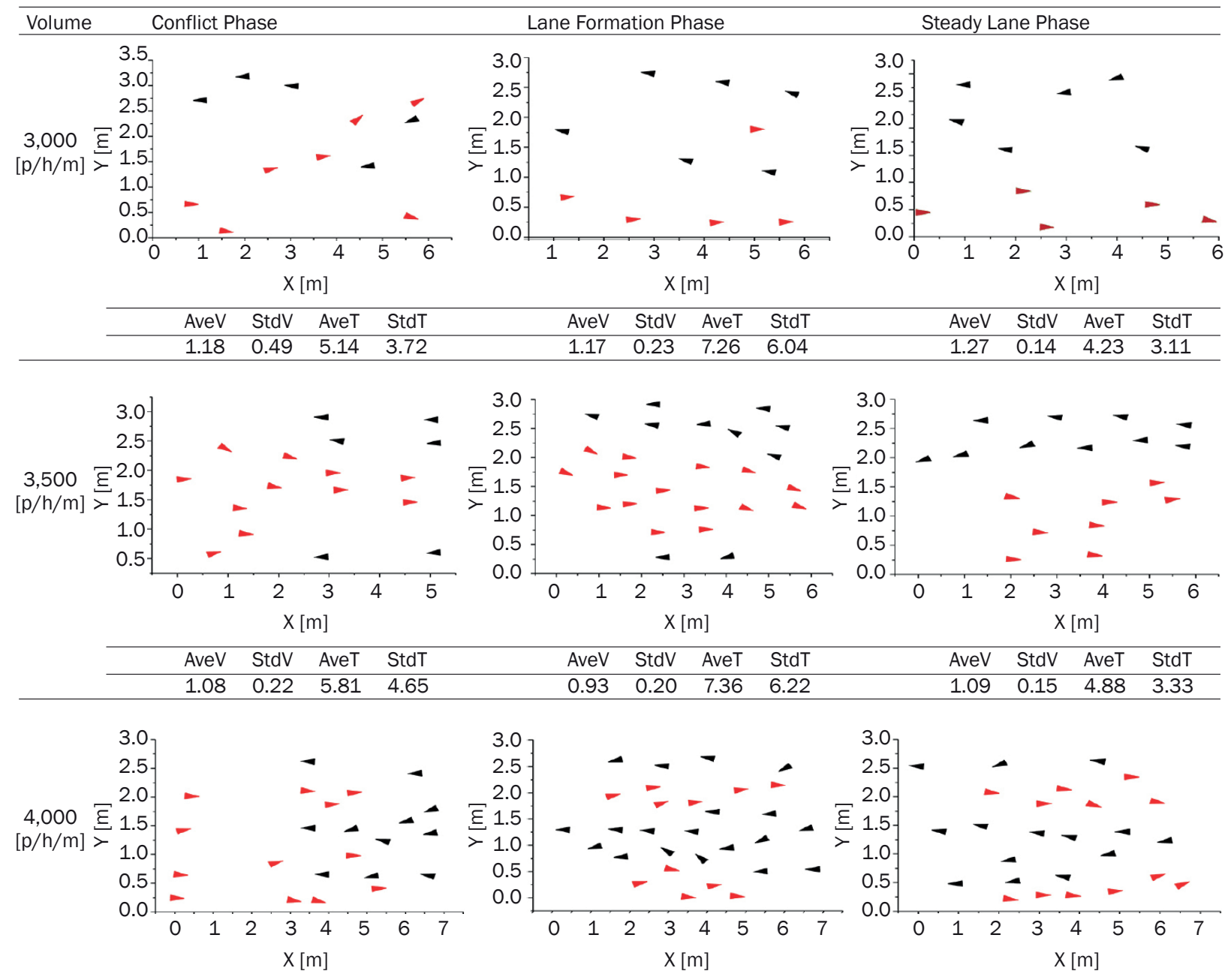

\begin{tabular}{cccccccccccc}
\hline AveV & StdV & AveT & StdT & AveV & StdV & AveT & StdT & AveV & StdV & AveT & StdT \\
\hline 1.1 & 0.27 & 7.13 & 5.47 & 0.98 & 0.26 & 9.18 & 7.86 & 1.18 & 0.17 & 5.47 & 4.08 \\
\hline
\end{tabular}

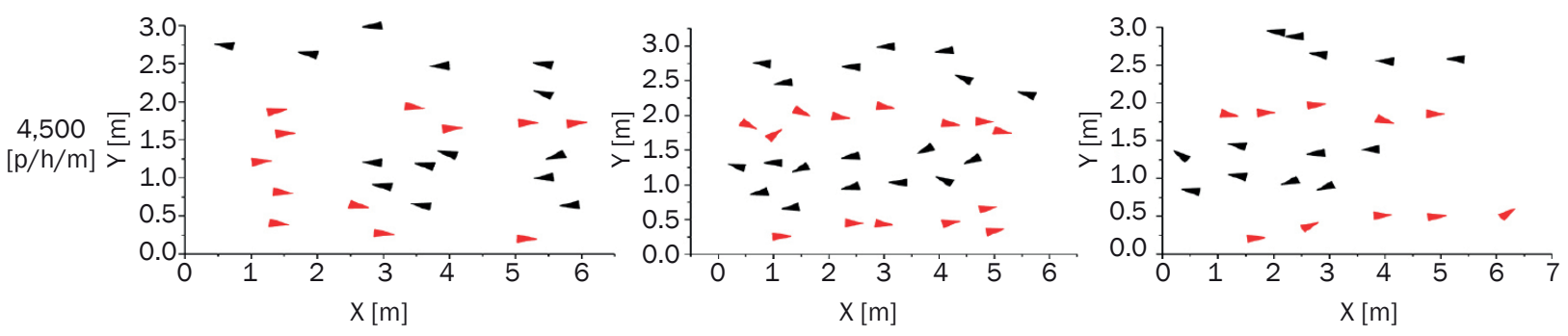

\begin{tabular}{rrrrrrrrrrrr}
\hline AveV & StdV & AveT & StdT & AveV & StdV & AveT & StdT & AveV & StdV & AveT & StdT \\
\hline 0.97 & 0.21 & 8.07 & 6.85 & 0.83 & 0.25 & 10.18 & 14.41 & 1.15 & 0.14 & 5.06 & 3.74 \\
\hline
\end{tabular}

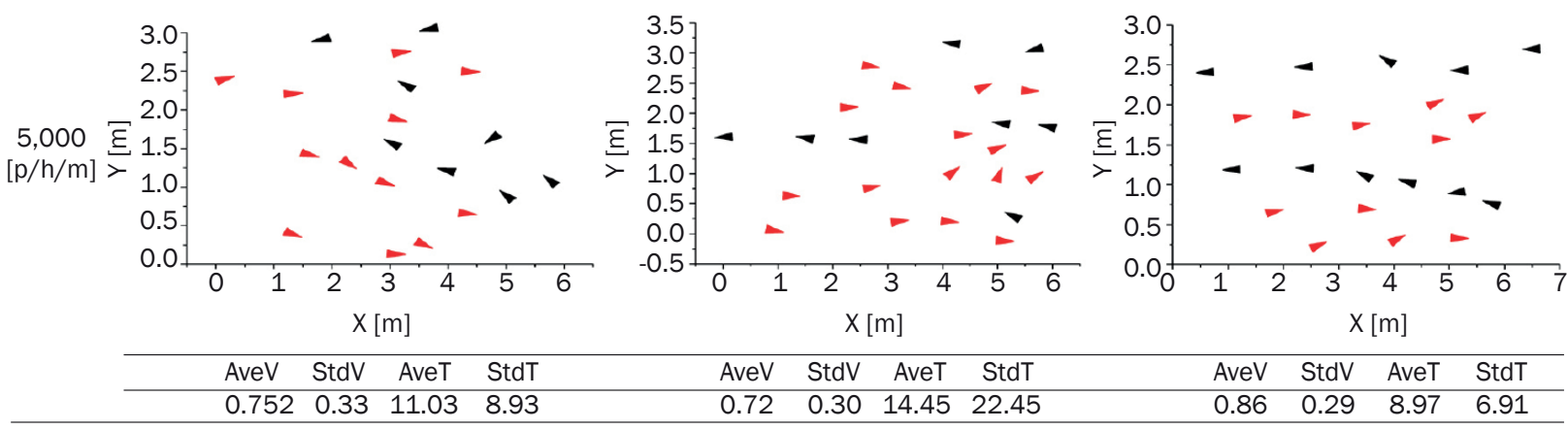

Figure 4 - Three phases of lane formation 


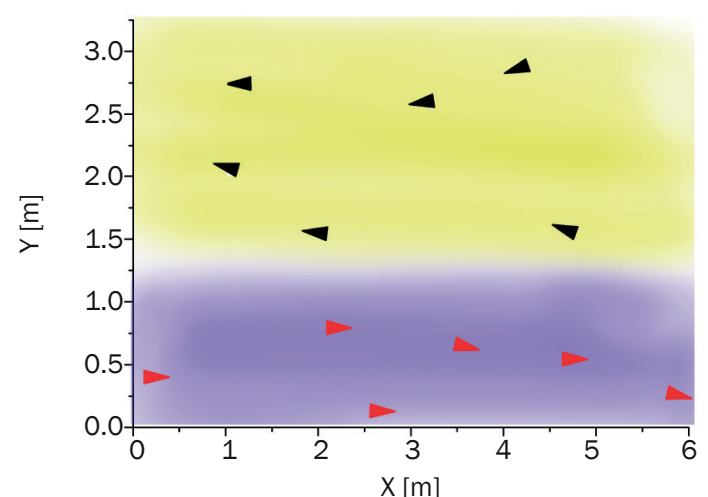

a) The steady lane under $3,000 \mathrm{p} / \mathrm{h} / \mathrm{m}$

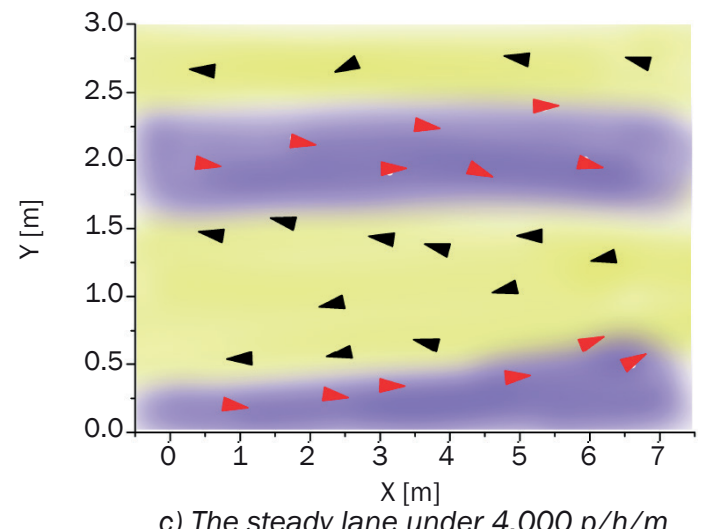

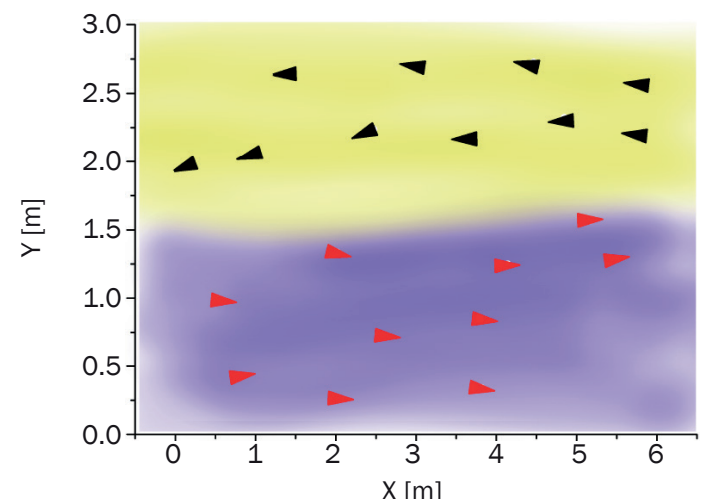

b) The steady lane under $3,500 \mathrm{p} / \mathrm{h} / \mathrm{m}$

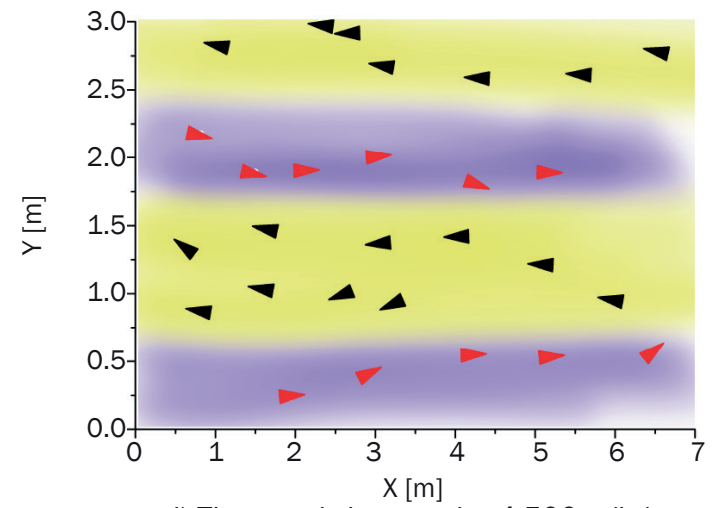

d) The steady lane under $4,500 \mathrm{p} / \mathrm{h} / \mathrm{m}$

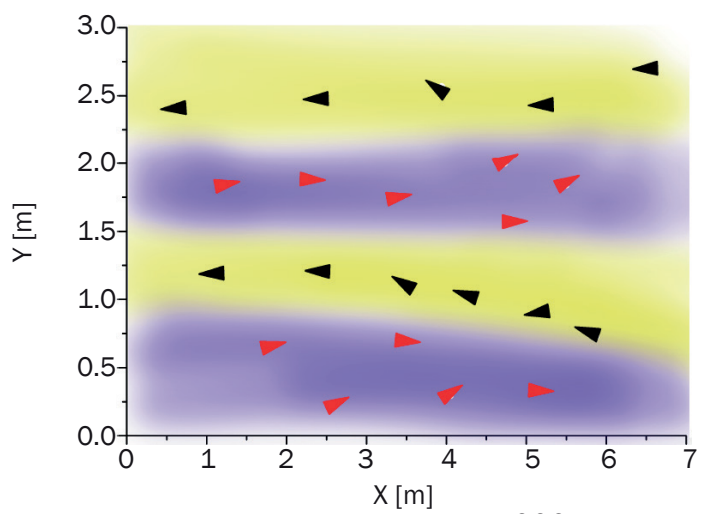

e) The steady lane under $5,000 \mathrm{p} / \mathrm{h} / \mathrm{m}$

Figure 5 - Steady lane in bidirectional flow experiment

The number of steady lanes was dependent on the volumes. For the scenarios of 3,000 p/h/m and 3,500 $\mathrm{p} / \mathrm{h} / \mathrm{m}$, only two lanes were formed. For the scenarios of more than $4,000 \mathrm{p} / \mathrm{h} / \mathrm{m}$ volume, four lanes were observed. Based on the theory of pedestrian traffic behaviour [17], the basic walking width of a single pedestrian is $0.75 \mathrm{~m}$. Experimental corridor width was only 3 $\mathrm{m}$, so four lanes were the maximum number of lanes observed that remain stable in the steady lane phase.

As shown in Figure 5, the distances between pedestrians in different layers were approximately constant and analogous. For the scenarios of $3,000 \mathrm{p} / \mathrm{h} / \mathrm{m}$ and $3,500 \mathrm{p} / \mathrm{h} / \mathrm{m}$, the average longitudinal distance between pedestrians (within a layer) was about 1.3-1.7 m.
For the scenarios of more than $4,000 \mathrm{p} / \mathrm{h} / \mathrm{m}$ or more, the longitudinal distance was $0.55-0.95 \mathrm{~m}$. Hence, the opposite directional lanes in the corridor can equally divide the corridor into two or four parts.

\section{COUNTERMEASURES FOR REDUCING CONFLICT}

The interesting discovery of the phenomenon of lane formation from the above study motivated the study of physically changing the layout of the corridor for reducing the traffic conflict. This section explained the effort of adding separating straps to the corridor based on the lane formation. In the corridor, a strap was placed from corridor entrance to exit to separate 
the corridor space. It was hypothesized that the system would form two regions for two groups of pedestrians moving in opposite directions and, thus, would yield the least friction between the two regions and the smallest collective disutility. Pedestrian experiments were conducted to validate the effectiveness of the countermeasures.

\subsection{Adding separating strap to subway corridor}

One option for strap placement is to add the separation between the adjacent lanes; i.e. putting the strap in the space between different direction pedestrian lanes. The other option is to add the separation without considering the formed lanes. This study considered and experimented with both options.

According to the former experiment results, the steady pedestrian lanes have equally divided the corridor into two or four parts under 1:1 flow ratio. Therefore, considering the possible position of the strap location, the suitable position for placing the strap on lane was placed in the centre of the corridor. Meanwhile, for the control group, adding the separation without considering the formed lanes were explored and a 1:2 ratio position was supposed to be suitable for the control group. In addition to the two scenarios of adding a strap, a no-strap placed scenario was set up as the base group. Table 5 lists three scenarios set up for analysis.

As set in the middle/third of the corridor to separate the corridor space, the strap did not interfere with the route layout of pedestrians, so that all participants could choose a path to the left or right of a strap according to their preferences.

\subsection{Parameter definition}

Both macroscopic and microscopic parameters were evaluated to compare the pedestrian conflict before and after the intervention. The microscopic parameters included the speed and the turning angle defined in the previous section; besides, the duration of each passenger travelling across the corridor was the other microscopic parameter [32-34].

Similar to the definition of the microscopic parameters, the two macroscopic parameters, the conflict point and the conflict area, were introduced to assess the extent of the conflict. In literature, the change of speed was used as the indicator to identify the conflict point [26]; if the speed at $t_{i+1}$ was reduced by a

Table 5 - Experiments setting percentage between $20 \%$ and $30 \%$ compared to the speed at $t_{i}$, the position of the passenger at $t_{i}$ was recognized as a conflict point [35-36]. This study used $30 \%$ as the threshold for the decrease of speed to determine a conflict point.

The total conflict point is an important indicator for analysing the conflict. A conflict centre is defined as the point that has minimum distance to all the other conflict points, calculated by Equation 2:

$$
\begin{aligned}
& z=\min \sum_{i=1}^{i=n} \sqrt{\left(x-x_{i}\right)^{2}+\left(y-y_{i}\right)^{2}} \\
& (i=1,2,3, \ldots n)
\end{aligned}
$$

where $z(x, y)$ is the coordinate of conflict centre and $\left(x_{i}, y_{i}\right)$ is the coordinate of conflict point $i$. A conflict area is an area dilated from the conflict centre and includes at least $85 \%$ of the conflict points. Referring to the regular statistic, the concept of $85^{\text {th }}$ percentage was adopted.

\subsection{Validation of the countermeasures}

Pedestrian experiments, which had the same group of participants and settings, were conducted for extracting the parameters for validating countermeasures.

\subsubsection{Pedestrian track analysis}

Figure 6 shows the variety of pedestrian speeds for three different scenarios, Scenario OL (add the separation between the adjacent lanes), Scenario NOL (add the separation without considering the formed lanes), and Scenario BA (no strap-placed scenario). Each line represents a pedestrian walking track. Colour gradients are used to represent the speed where black colour indicates the lowest while grey means the highest.

Scenario OL had the best performance among the three scenarios; its overall speed was close to a free flow speed, and its speed variation was small, as shown in Figure 6a. For both Scenario NOL and Scenario $\mathrm{BA}$, the conflicts were evident from the varied lines, and some black spots were even observed.

Lane formation and passenger conflict were further investigated by examining the video footages. For Scenario OL, mainly two lanes were formed, the twoway traffic was mostly separated by the strap, and the conflict mostly concentrated in the areas near the

\begin{tabular}{||l|l|c||}
\hline Scenario & Countermeasure & Location of strap \\
\hline \hline On the lane $(\mathrm{OL})$ & add the separation between the adjacent lanes & $1: 1$ \\
\hline Not on the lane $(\mathrm{NOL})$ & add the separation without considering the formed lanes & $1: 2$ \\
\hline Base $(\mathrm{BA})$ & no strap placed scenario & none \\
\hline
\end{tabular}



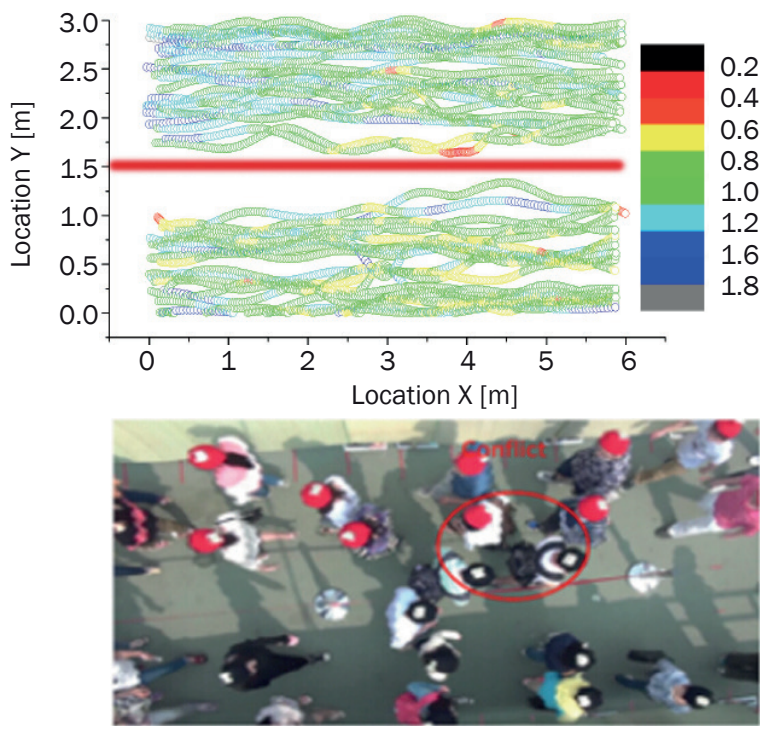

a) Scenario $\mathrm{OL}$
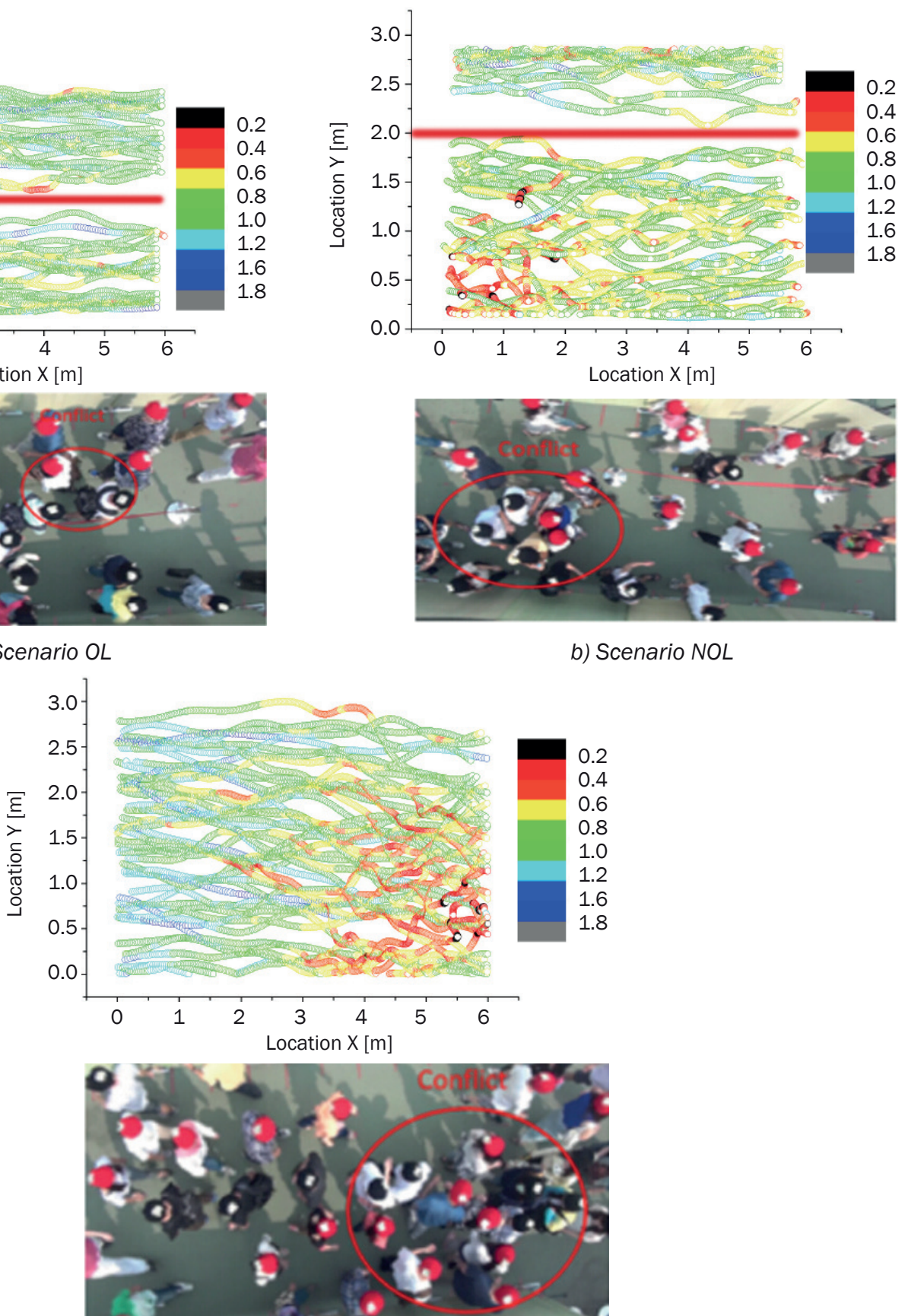

c) Scenario BA

Figure 6 - Pedestrian speed and conflict for three validation scenarios

strap where a few pedestrians walked against the major flow. Since the participants had the arbitral selection of either side of the strap, similar to a real-world situation, the conflicts occurred from different directions on the same side of the strap. Because of the small number, the conflicts were low to moderate. This conforms to the fact that a majority of Chinese people are right-hand dominated and are inclined to walk on the right side. For Scenario NOL and Scenario BA, however, the lanes were irregular, and the conflicts were severe.

\subsubsection{Speed, turning angle, and travel time analysis}

Table 6 lists the statistical data, extracted from the validation experiments, to analyse the impact of the layout of the corridor on passenger flow. Considering the speed, turning angle, and travel time measures, Scenario OL had the best performance, whereas Scenario BA had the worst. The average speed, turning angle, and travel time of Scenario OL were better than those of Scenario BA by $22.2 \%, 28.5 \%$, and $20.7 \%$, respectively. This significant improvement indicated that 
Table 6 - Microscopic pedestrian performance parameters for different scenarios

\begin{tabular}{||c|l|c|c|c|c|c||}
\hline \multicolumn{1}{|c|}{ Index } & \multicolumn{1}{|c|}{ Position of corridor } & Max & Min & Median & Mean & Std. \\
\hline \hline \multirow{3}{*}{ Speed/[m/s] } & Scenario OL & 1.874 & 0.396 & 0.993 & 1.023 & 0.215 \\
\cline { 2 - 7 } & Scenario NOL & 1.781 & 0.011 & 0.813 & 0.854 & 0.235 \\
\cline { 2 - 8 } & Scenario BA & 1.768 & 0.008 & 0.841 & 0.837 & 0.309 \\
\hline \multirow{4}{*}{ Turning angle/ $\left.{ }^{\circ}\right]$} & Scenario OL & 139.531 & 0.027 & 5.356 & 6.84 & 7.765 \\
\cline { 2 - 8 } & Scenario NOL & 167.381 & 0.009 & 8.286 & 8.163 & 11.367 \\
\cline { 2 - 8 } & Scenario BA & 175.433 & 0.006 & 6.84 & 9.561 & 12.529 \\
\hline \multirow{3}{*}{ Travel time/[s] } & Scenario OL & 5.22 & 2.82 & 4.02 & 4.146 & 0.654 \\
\cline { 2 - 8 } & Scenario NOL & 6.82 & 3.22 & 4.82 & 5.017 & 0.842 \\
\cline { 2 - 8 } & Scenario BA & 8.02 & 3.42 & 5.32 & 5.228 & 1.173 \\
\hline \hline
\end{tabular}

placing the strap on the lane is an effective countermeasure to reduce the pedestrian conflict. However, the average speed, turning angle, and travel time of Scenario NOL was better than those of Scenario BA only by $2.0 \%, 14.6 \%$, and $4.0 \%$, respectively, which implies that if the separating strap was not placed on the lane, the effectiveness was compromised.

\subsubsection{Conflict point and conflict area analysis}

Table 7 lists the conflict point and conflict area from the three scenarios. Scenario OL had 120 conflict points and $9.08 \mathrm{~m}^{2}$ conflict area, about $25 \%$ less than the Scenario BA. The conflict points of Scenario NOL were significantly increased compared to the ones of Scenario BA; this echoed the previous finding that the separation should be placed on the lane.

Table 7 - Statistics for conflict point and conflict area

\begin{tabular}{||l|c|c||}
\hline Position of corridor & Conflict point & $\begin{array}{c}\text { Conflict area } \\
{\left[\mathrm{m}^{2}\right]}\end{array}$ \\
\hline \hline Scenario OL & 120 & 9.08 \\
\hline Scenario NOL & 226 & 10.03 \\
\hline Scenario BA & 157 & 11.88 \\
\hline
\end{tabular}

\section{DISCUSSION AND RECOMMENDATIONS}

The design of infrastructure, as well as the assessment of its efficiency and safety, requires the investigation of pedestrian behaviours under bidirectional flow. Previous studies on this topic relied on computer simulations [7-9], which built a simulated model based on patterns of pedestrian behaviours through the method of the cellular automaton or the social force [37-38]. The studies using computer simulations considered the behaviour of right-hand-sided, lane changing, and the collision avoidance in the complex bidirectional flow [39, 40]; they had shortcomings, including poor randomness, ignoring diversity and adaptability of pedestrian, and poor simulation accuracy. Collecting data in the rail station, although preferable because of the fidelity, is unfeasible because of the complex conditions, such as low ceilings, uncontrollable traffic volume, and multiple mixed pedestrian flows. This study used an alternative approach, conducting experiments to simulate a real situation in rail station [19-20], to analyse pedestrian behaviours.

After lane formation was recognized in bidirectional flow $[4,8,20]$, literature qualitatively explored the process of lane formation including conflict, following effect [39] and evasive effect [41, 42]. When the pedestrian volume of the bi-bidirectional flow increases, the lane formation has three patterns that are a free pattern, a lanes pattern and a jam pattern with the increase of volumes [43]. The average speed decreases with the increase of volume in the whole system [44]. The literature mentioned the above concentrated mainly on the macroscopic characteristics of the bidirectional flow but did not involve the microscope characteristics related to individual pedestrians. This motivated us to study quantitatively the evolution mechanism of the lane formation process under the microscope parameters, such as the speed and the turning angle, both of which were time-dependent. This also motivated us to study the inherent relations between the pedestrian behaviours and the microscope parameters. It was hypothesized that individual's microscope parameters represented the actual characteristics of the pedestrian behaviour [40].

Also, this study investigated the characteristics of lane formation. Helbing [45] analysed the characteristics of lane and proposed that the number of formed lanes depended on the density in the walking area. Hoogendoorn and Daamen [11] studied it quantitatively by cluster analysis and discovered patterns of two, three, and four lanes. Kretz et al. [21] found that an odd number of lanes was likely to break the symmetry. Most studies on the lane characteristics considered the whole process of lane formation and ignored the fact that lanes would disappear, merge, split and be stable. In this study, it was found that the pedestrian lanes were stable if their number was even (two or four) and that a steady number of lanes was 
dependent on the pedestrian volume, e.g. two lanes appeared at the pedestrian volume less than 4,000 $\mathrm{p} / \mathrm{h} / \mathrm{m}$ and four lanes appeared at the one more than $4,000 \mathrm{p} / \mathrm{h} / \mathrm{m}$. It was also found that the width of the lane was approximately constant and analogous (the width of the two lanes was $1.3-1.7 \mathrm{~m}$, and the width of the four lanes was $0.55-0.95 \mathrm{~m}$ ).

This study was the first to use countermeasures based on the characteristics of the lane formation to examine the method for reducing the conflicts of pedestrian flows. The before-and-after analysis was applied to demonstrate the effectiveness of the different countermeasures. The results showed that adding the separation between the adjacent lanes had the best performance in reducing the conflicts.

As a deficiency, this study only considered the flow ratio $1: 1$ and a single environment. Future studies will use different flow ratios and different widths of corridors.

\section{CONCLUSION}

Bidirectional flow is one of the most commonly seen passenger organization forms in Beijing subway corridor. This study explored the characteristics and countermeasures of the bidirectional pedestrian flow of 1:1 flow ratio in subway corridor. Pedestrian experiments were conducted to investigate microscopic characteristics of the pedestrian flow. It was found that the microscopic characteristics, including the walking speed and turning angle, were time-dependent and had a generalized trend through the experimental period. It was also found that the different pedestrian volumes affected the microscopic characteristics. Based on the trend of the microscopic characteristics, the lane formation phenomenon was observed and quantitatively studied, identifying three phases: conflict phase, lane formation phase, and steady lane phase. To alleviate the bidirectional pedestrian conflict, additional pedestrian experiments for the countermeasure of adding separating strap in the corridor, which was based on the lane formation analysis, was conducted. The effectiveness of the countermeasure was demonstrated through a before-and-after comparison. The results showed that adding the separation between the adjacent lanes had the best performance in reducing the conflicts. The results would provide a rationale for subway managers in optimizing the corridor bidirectional pedestrian flow.

\section{ACKNOWLEDGEMENT}

The authors would like to acknowledge the financial support for this study provided by the National Natural Science Foundation of China (No. 51308017), Beijing Nova Program (Grant No. Z141106001814110), Funding Project for Academic Human Resources Development in Institutions of Higher Learning under the
Jurisdiction of Beijing Municipality and Science and Technology Program of Beijing (D161100005616001).

\section{乔婧 孙立山 邱实 荣建 刘小明}

\section{基于分层特征的轨道交通双向行人流冲突优化研究}

摘要: 随着轨道交通客运量迅速增加, 客流冲突特 别是双向客流冲突日益成为影响地铁运营的重要问 题。本研究以行人实验为手段, 解析行人流动的微 观特征, 研究发现速度和转角随时间增长而增加趋 势且不同流量下微观特性不同。进而定量研究行人 流分层过程, 提出冲突、层形成和层稳定三个阶段 并深度解析分层特征。为缓解双向行人冲突, 考虑 基于层形成特性增设通道护栏, 利用行人实验对比 论证了对策的有效性。结果表明, 在层间设置护栏 具有最好的性能, 可有效降低冲突。本研究可为地 铁通道双向行人流组织优化提供科学依据。

关键词：轨道交通, 正向行人流, 行人实验, 管理 措施

\section{REFERENCES}

[1] Beijing Municipal Transportation Operations Coordination Center. Beijing transport annual report. Volume 1. Beijing: Beijing Municipal Commission of Transport Publishing Service; 2013.

[2] Campanella M, Hoogendoorn SP, Daamen W. Effects of heterogeneity on self-organized pedestrian flows. Transportation Research Record:Journal of the Transportation Research Board. 2009;2124(1): 148-156. doi: 10.3141/2124-14

[3] Johansson A. Constant-net-time headway as a key mechanism behind pedestrian flow dynamics. Physical Review E. 2009;80(2): 1-7. doi: 10.1103/PhysRevE.80.026120

[4] Seyfried A, Passon O, Steffen B, Boltes M, Rupprecht T, Klingsch W. New insights into pedestrian flow through bottlenecks. Transportation Science. 2009;43(3): 395-406. doi: 10.1287/trsc.1090.0263

[5] Older SJ. Movement of pedestrians on footways in shopping streets. Traffic engineering \& control. 1968;10(4): 160-163.

[6] Helbing D, Farkas IJ, Molnar P, Vicsek T. Simulation of pedestrian crowds in normal and evacuation situations. International Conference on Pedestrian and Evacuation Dynamics; 2001 APR 04-06; Duisburg, Germany. Berlin: Springer; 2002.

[7] Guo RY, Wong SC, Huang HJ, Zhang P, Lam WH. A microscopic pedestrian-simulation model and its application to intersecting flows. Physica A: Statistical Mechanics and its Applications. 2010;389(3): 515-526. doi: 10.1016/j.physa.2009.10.008

[8] Xie S, Wong SC, Lam WH, Chen A. Development of a bidirectional pedestrian stream model with an oblique intersecting angle. Journal of Transportation Engineering. 2013;139(7): 678-685. doi: 10.1061/(ASCE) TE.1943-5436.0000555

[9] Blue V, Adler J. Bi-directional emergent fundamental pedestrian flows from cellular automata microsimulation. Transportation and Traffic Theory. 1999;14: 235-254. 
[10] Blue VJ, Adler JL. Cellular automata microsimulation for modeling bi-directional pedestrian walkways. Transportation Research Part B: Methodological. 2001;35(3): 293-312. doi: 10.1016/S0191-2615(99)00052-1

[11] Hoogendoorn S, Daamen W. Self-organization in Pedestrian Flow, In: Hoogendoorn S, Luding S, Bovy P, et al. (eds.) Traffic and Granular Flow '03. Berlin: Springer-Verlag, 2005; p. 373-382.

[12] Zhang J, Song W, Xu X. Experiment and multi-grid modeling of evacuation from a classroom. Physica A: Statistical Mechanics and its Applications. 2008;387(23): 5901-5909. doi: 10.1016/j.physa.2008.06.030

[13] Chertock A, Kurganov A, Polizzi A, Timofeyev I. Pedestrian flow models with slowdown interactions. Mathematical Models and Methods in Applied Sciences. 2014;24(2):249-275. doi: 10.1142/ S0218202513400083

[14] Hughes RL. The flow of large crowds of pedestrians. $3^{\text {rd }}$ IMACS Symposium on Mathematical Modelling; 2000 FEB 02-04; Vienna, Austria. Amsterdam: Elsevier; 2000.

[15] Hughes RL. A continuum theory for the flow of pedestrians. Transportation Research Part B: Methodological. 2002;36(6): 507-535. doi: 10.1016/S01912615(01)00015-7

[16] Helbing D. A stochastic behavioral model and a 'microscopic' foundation of evolutionary game theory. Theory and Decision. 1996;40(2): 149-179.

[17] Lam WH, Lee JY, Cheung CY. A study of the bi-directional pedestrian flow characteristics at Hong Kong signalized crosswalk facilities. Transportation. 2002;29(2): 169-192. doi: 10.1023/A:1014226416702

[18] Lam WH, Lee JY, Chan KS, Goh PK. A generalised function for modeling bi-directional flow effects on indoor walkways in Hong Kong. Transportation Research Part A: Policy and Practice. 2003;37(9): 789-810. doi: 10.1016/S0965-8564(03)00058-2

[19] Daamen W, Hoogendoorn SP. Experimental research of pedestrian walking behaviour. $82^{\text {nd }}$ Annual Meeting of the Transportation-Research-Board; 2003 JAN 1216. Washington, D.C. USA.

[20] Helbing D, Buzna L, Johansson A, Werner T. Self-organized pedestrian crowd dynamics: Experiments, simulations, and design solutions. Transportation Science. 2005;39(1): 1-24. doi: 10.1287/trsc.1040.0108

[21] Kretz T, Grünebohm A, Kaufman M, Mazur F, Schreckenberg M. Experimental study of pedestrian counterflow in a corridor. Journal of Statistical Mechanics: Theory and Experiment. 2006;2006(10): 1-22. doi: 10.1088/1742-5468/2006/10/P10001

[22] Zhang J, Klingsch W, Schadschneider A, Seyfried A. Ordering in bidirectional pedestrian flows and its influence on the fundamental diagram. Journal of Statistical Mechanics: Theory and Experiment. 2012;P02002(02): 1-20. doi: 10.1088/1742-5468/2012/02/P02002

[23] Golas A, Narain R, Curtis S, Lin MC. Hybrid long-range collision avoidance for crowd simulation. IEEE Transactions on Visualization and Computer Graphics. 2014;20(7): 1022-1034. doi: 10.1109/TVCG.2013.235

[24] Transit Cooperative Research Program. Transit Capacity and Quality of Service Manual. Volume 7. Washington DC: Transportation Research Board of the National Academies Publishing Service; 2003.
[25] Sun L, Luo W, Yao LY, Qiu S, Rong J. A comparative study of funnel shape bottlenecks in subway stations. Transportation Research Part A: Policy \& Practice. 2017;98: 14-27.

[26] Sun L, Yang Z, Rong J, Liu XM. Study on the Weaving Behaviour of High Density Bidirectional Pedestrian Flow. Mathematical Problems in Engineering, 2014;2014: 1-9. doi: $10.1155 / 2014 / 765659$

[27] Stauffer C, Grimson WEL. Learning patterns of activity using real-time tracking. IEEE Transactions on Pattern Analysis \& Machine Intelligence. 2000;22(8): 747-757. doi: 10.1109/34.868677

[28] Piccoli B, Tosin A. Pedestrian flows in bounded domains with obstacles. Continuum Mechanics and Thermodynamics. 2009;21(2): 85-107. doi: 10.1007/ s00161-009-0100-x

[29] Cancho RFI, Solé RV. Least effort and the origins of scaling in human language. Proceedings of the National Academy of Sciences. 2003;100(3): 788-791. doi: 10.1073/pnas.0335980100

[30] Johansson A, Helbing D, Al-Abideen HZ, Al-Bosta S. From crowd dynamics to crowd safety: a video-based analysis. Advances in Complex Systems. 2008;11(04): 497-527. doi: 10.1142/S0219525908001854

[31] Cristiani E, Piccoli B, Tosin A. Multiscale modeling of granular flows with application to crowd dynamics. Multiscale Modeling \& Simulation. 2011;9(1): 155-182. doi: 10.1137/100797515

[32] Antonini G, Bierlaire M, Weber M. Discrete choice models of pedestrian walking behaviour. Transportation Research Part B: Methodological. 2006;40(8): 667687. doi: 10.1016/j.trb.2005.09.006

[33] Teknomo K. Application of microscopic pedestrian simulation model. Transportation Research Part F: Traffic Psychology and Behaviour. 2006;9(1): 15-27.

[34] Sarmady S, Haron F, Talib AZ. A cellular automata model for circular movements of pedestrians during Tawaf. Simulation Modelling Practice and Theory. 2011;19(3): 969-985. doi: 10.1016/j.simpat.2010.12.004

[35] Sano T, Shida K, Tatebe K. An experimental study on pedestrian crossing conflicts by physical index: Study on characteristics of pedestrian crossing flow. Journal of Architecture Planning \& Environmental Engineering. 2001;(8): 127-132.

[36] Daamen W, Hoogendoorn S. Calibration of pedestrian simulation model for emergency doors by pedestrian type. Transportation Research Record: Journal of the Transportation Research Board. 2012;2316(1): 69-75. doi: 10.3141/2316-08

[37] WengWG, Shen SF, Yuan HY, Fan WC. A behaviour-based model for pedestrian counter flow. Physica A Statistical Mechanics \& Its Applications. 2007;375(2): 668-678. doi: 10.1016/j.physa.2006.09.028

[38] Li MH, Yuan ZZ, XU Y, Tian JF. Randomness analysis of lane formation in pedestrian counter flow based on improved lattice gas model. Acta Physica Sinica. 2015;64(1): 018903. doi: 10.7498/aps.64.018903

[39] Lee J, Kim T, Chung JH, Kim J. Modeling lane formation in pedestrian counter flow and its effect on capacity. KSCE Journal of Civil Engineering. 2016;20(3): 10991108. doi: 10.1007/s12205-016-0741-9

[40] Kretz T, Kaufman M, Schreckenberg M. Counterflow Extension for the FAST-Model. $8^{\text {th }}$ International 
Conference on Cellular Automata for Research and Industry; 2008 Sep 23-26. Yokohama, Japan.

[41] Alonsomarroquin F, Lozano C, Ramirezgomez A, Busch J. Simulation of counter flow pedestrian dynamics in hallways using spheropolygons. Physical Review E: Statistical Nonlinear \& Soft Matter Physics. 2013;90(6): 063305. doi: 10.1103/PhysRevE.90.063305

[42] Xiong T, Zhang P, Wong SC, Shu CW, Zhang MP. A macroscopic approach to the lane formation phenomenon in pedestrian counterflow. Chinese Physics Letters. 2011;28(10): 108901. doi: 10.1088/0256-307X/28/10/108901
[43] Guo W, Wang X, Zheng X. Lane formation in pedestrian counterflows driven by a potential field considering following and avoidance behaviours. Physica A: Statistical Mechanics \& Its Applications. 2015;432: 87-101. doi: 10.1016/j.physa.2015.03.020

[44] Nowak S, Schadschneider A. Quantitative analysis of pedestrian counterflow in a cellular automaton model. Physical Review E: Statistical Physics Plasmas Fluids \& Related Interdisciplinary Topics. 2012;85(2): 482-510. doi: 10.1103/PhysRevE.85.066128

[45] Helbing D. Traffic Dynamics: New Physical Modeling Concepts. Berlin: Springer-Verlag; 1997. 